\title{
Calculation of measurement uncertainty for plastic (ABS) material in flexural testing
}

\author{
A. Gunay ${ }^{1, \star}$, S. Fank ${ }^{2}$, T. Gulmez ${ }^{3}$, and N.M. Durakbasa ${ }^{4}$ \\ 1 Yildiz Technical University, Mechanical Engineering Department, Istanbul, Turkey \\ 2 The Scientific and Technical Research Council of Turkey, National Metrology Institute (TUBITAK-UME), Force Group \\ Laboratory, Kocaeli, Turkey \\ 3 Istanbul Technical University, Mechanical Engineering Department, Istanbul, Turkey \\ 4 Vienna University of Technology, Institute for Production Engineering and Laser Technology, Department Interchangeable \\ Manufacturing and Industrial Metrology, Vienna, Austria
}

Received: 31 October 2012 / Accepted: 22 November 2012

\begin{abstract}
In order to determine mechanical properties of materials various kind of tests can be applied by means of using their tensile strength, lower yield stress, proof stress, impact strength, Brinell, Rockwell and surface hardness, elongation after fracture properties. Among these tests, three point flexural testing method has some advantages such as easy preparation (production) of samples and no gripping problems comparing to tension test. Flexural tests results should be obtained accurately to provide expected testing performance. The measurement uncertainty of flexural tests should be calculated by conducting all effective uncertainty parameters during the test procedure. In this study, the measurement uncertainty of the flexural test of ABS (Acrylonitrile Butadiene Styrene) material was investigated, which is widely used as industrial plastic material in many applications.
\end{abstract}

Keywords: Uncertainty of flexural testing; three point bending test; ABS (Acrylonitrile Butadiene Styrene) material

\section{Introduction}

In prior to improve their product quality and become more powerful in today's competitive industrial market, significant amount of companies are making mechanical tests on the products materials to develop their properties. Newly developed materials also need attention for developing their measurement techniques accuracy that will reduce the costs and manufacturing, marketing time. Knowing material properties accurately will help the producer choosing better material and more appropriate manufacturing parameters to meet better quality of their products. This will prevented a possible damage in use. The flexural test is based on the application of bending force of the materials. As in many other flexural test methods, 3 point bending test method also has similar principle; material is loaded to find the relationship between load and elongation of materials. Three point bending test can be called shortly; this testing method measures the force required to bend a beam under 3 point loading conditions.

Uncertainty and accuracy of measurement results have a vital importance for selection of material which meets the requests [1]. As a result of investigations is that the length between dies the most effective parameter. In this

^ Correspondence: gunay@yildiz.edu.com.tr study the effect of different between the pins to the uncertainty was investigated.

As result of the research, conclusions can be drawn about the correlation of measurement uncertainty between different specimen lengths with the overall goal to achieve lower costs and higher quality in measurement [2].

\section{Material and method}

The three-point bending is a conventional testing method that is analyzed taking into account shear and local deformation effects in the load application and supports. This test method is using widely for the determination of mechanical properties of materials especially subjecting to bending force. Three point bending test has also an advantage as a flexural test that sample geometries are easy to produce and there are no gripping problems can occur in tensile tests [3]. In addition to these, the results can be easily calculated and the test is easy to apply.

In this experimental study sample materials were Acrylonitrile Butadiene Styrene (ABS). ABS is the most common of industrial plastics and they are generally produced by injection mouldings. Injection molded products cannot be straight in cross section, thickness at the centre 
Table 1. Sample dimensions.

\begin{tabular}{cccccccc}
\hline Samples & $\begin{array}{c}\text { Nominal thickness } \\
h\end{array}$ & $\begin{array}{c}\text { Width } \\
b\end{array}$ & $R_{\mathbf{1}}$ & $R_{\mathbf{2}}$ & $L$ & $l$ & Samples \\
\hline ABS-1 & 2.82 & 10 & 5.02 & 5.08 & 62 & 110 & ABS-1 \\
\hline ABS-2 & 3.69 & 25 & 5.01 & 2.12 & 47 & 75 & ABS-2 \\
\hline
\end{tabular}

of the samples measured as the smallest value of the thickness [4]. The difference between the smallest $h$ and the maximum $h$ values were changed between $0.1 \sim 0.06 \mathrm{~mm}$.

The thicknesses of the samples were measured from ten different random points place along the length. The thinner specimens had a length of $75 \mathrm{~mm}$ and a width of $25 \mathrm{~mm}$. The distance between the supports, $L$ was calculated as $48 \mathrm{~mm}, 62 \mathrm{~mm}$. The crosshead speed was selected as $1 \mathrm{~mm} / \mathrm{min}$ from ISO 178 [5]. Twenty different specimens were tested under same conditions to reduce uncontrolled uncertainty sources effect like inhomogeneous internal structure of molded material.

\section{Uncertainty analyses}

As in the ISO/IEC Guide 99:2007 [6] stated and in [7] mentioned: "Traceability is the property of the result of a measurement whereby it can be related to stated references, usually national or international standards, through a documented unbroken chain of comparisons all having stated uncertainties".

From this definition the importance of uncertainty and accuracy of the procedure in usage area can be stated.

There are many different kind uncertainty sources. Some of them are expected and can be monitored. But also there are unexpected sources as known. Including all of the uncertainty components as seen means a really strength measurement output. At the same time, including these all components mean more tasks and heavier numerical computation at the formulation step.

In the calculation step repetitive measurements were performed in order to prevent unexpected errors. Then main uncertainty sources considered. Tensile force testing machine calibration certificate checked and measurement uncertainty quantity noticed. Caliper was also checked which was used to control sample dimensions. Then, the standard deviation of the uncertainty of a tensile strength measurement is calculated by means of 20 repeated test results. Combined standard uncertainty estimated by using sum of squares computational methods. Each corresponding sensitivity coefficients squares multiplied for determining combined standard uncertainty $U_{c}$ according to the standard:

$$
u_{c}=\sqrt{\sum c_{i}^{2} u_{i}^{2}}
$$

$u_{i}$ : uncertainty contributors;

$c_{i}$ : sensitivity coefficients.

The sensitivity coefficient $c_{i}$ of the input quantity $x_{i}$ was given by which expresses mathematically how much $f$

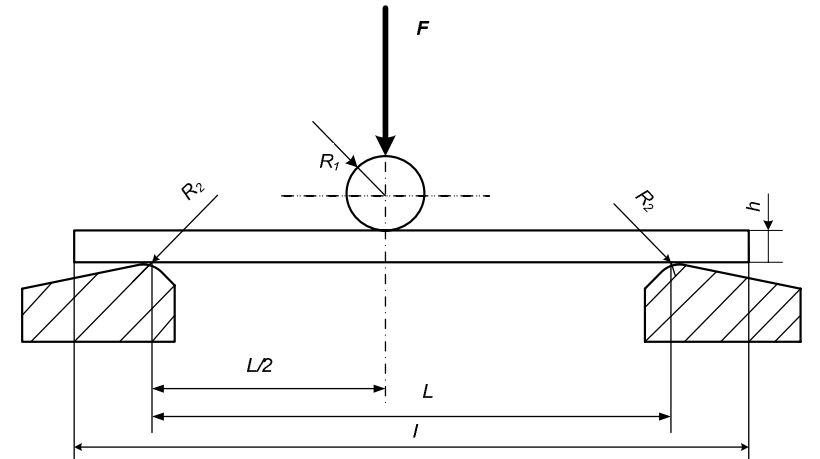

Fig. 1. Test set-up according to ISO 178 [4].

changes was given an infinitesimal change in $x_{i}[8,9]$ :

$$
c_{i}=\frac{\partial f}{\partial c_{i}}
$$

The data taken from experiments were used in order to get uncertainty. Firstly bending modulus was calculated according to formula for 3 point bending test according to Figure 1:

$$
\begin{aligned}
E_{b 3} & =\frac{l_{1}^{3}\left(F_{2}-F_{1}\right)}{4 b t^{3}\left(a_{2}-a_{1}\right)} \\
\sigma_{f} & =\frac{3 F I}{8 b h^{2}}
\end{aligned}
$$

$l$ : the length between outer dies, $\mathrm{mm}$

$b$ : width of the specimen, $\mathrm{mm}$

$t$ : thickness of the specimen, mm

$F_{2}-F_{1}$ : difference of load-deflection curve, $N$

$a_{1}-a_{2}$ : deflection at the center of load length current work and gives prospects for the future research.

$$
\sigma_{f}=\frac{1.5 F L}{b h^{2}}
$$

In equation (5), the flexural stress $\left(\sigma_{f}\right)$ was calculated using applied force $(F)$, length between two support point $(L)$ and sample dimensions $(b$ and $h)$.

Partial derivates of equations (2) and (4) were taken according to each parameter given in formula; sensitivity coefficients $c_{F}, c_{l}, c_{b}, c_{h}, c_{t}, c_{a}, c_{F}, c_{L}, c_{b}$ and $c_{h}$ were calculated easily. The measurement uncertainties of $\left(U_{E b 3}\right)$ and $\left(U_{\sigma f}\right)$ were calculated as given equations (6) and (7).

$$
\begin{aligned}
& u_{E_{b 2}}=\sqrt{c_{F}^{2} u_{F}^{2}+c_{1}^{2} u_{1}^{2}+c_{b}^{2} u_{b}^{2}+c_{h}^{2} u_{h}^{2}+c_{t}^{2} u_{t}^{2}+c_{a}^{2} u_{a}^{2}} \\
& u_{E_{b 2}}=\sqrt{c_{F}^{2} u_{F}^{2}+c_{L}^{2} u_{L}^{2}+c_{b}^{2} u_{b}^{2}+c_{h}^{2} u_{h}^{2}}
\end{aligned}
$$


$c_{F}:$ sensitivity coefficient for applied force measurement;

$c_{a}$ : sensitivity coefficient for cross-section measurement of test bar;

$u_{F}$ : measurement uncertainty of applied force taken directly from calibration certificate of material testing machine;

$u_{a}$ : measurement uncertainty of cross-section of the test bar.

Measurement uncertainties $u_{F}, u_{l}, u_{b}, u_{h}, u_{t}, u_{a}, u_{L}$ were calculated using directly measured test results.

In some measurements, statistical method can also be used for observed test results. The test data covers the influencing parameters. The standard deviation of test data gives the standard uncertainty of testing $\left(u_{\text {test }}\right)$ as a combined uncertainty of the above error sources in equation (7):

$$
u_{\text {test }}=\frac{s_{\text {test }}}{\sqrt{n_{\text {test }}}}
$$

$s_{\text {test }}$ : standard deviation of the test results of five test bars for tensile strength;

$n_{\text {test }}$ : number of test results for tested bars.

$$
s_{\text {test }}=\sqrt{\frac{\sum_{i=1}^{n}\left(X_{i} \check{S} \bar{X}_{t e s t}\right)^{2}}{n_{\text {test }} \check{S} 1}}
$$

$X_{i}$ : value of the flexural strength measurement;

$\overline{X_{i}}$ : average of the flexural strength measurement.

With these values combined measurement uncertainty was computed by using flexural strength $\left(u_{\sigma f}\right)$ and test uncertainty $\left(u_{\text {test }}\right)$. The calculation of measurement uncertainty for flexural testing as shown in below

$$
U_{\text {combined }}=\sqrt{u_{\sigma_{f}}^{2}+u_{\text {test }}^{2}} .
$$

There are many different kind uncertainty sources like

- Definition of the characteristics.

- Measuring procedure. The method of gripping the test piece and axiality of the application of the force, deflection errors (Fig. 4).

- Physical constants.

- Software and calculations of the tensile properties.

- Operator.

- Measurement environment like test temperature and loading rates in successive stages of tests.

- Software and calculations.

- Measuring object and reference element. The material in homogeneity that exists even within a single processed batch obtained from a single melt of material.

Including all of the uncertainty components as seen in Figure 2 means a really strength measurement output. At the same time, including these all components mean more tasks and heavier numerical computation at the formulation step.

To make this computation easier expanded uncertainty is used. Estimated expanded uncertainty $\left(U_{\exp }\right)$ was calculated as

$$
U_{\exp }=k U_{\text {combined }} \text {. }
$$

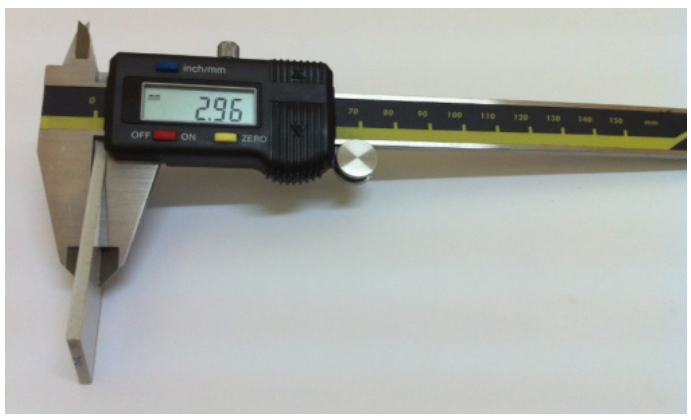

Fig. 2. Measurement of test sample dimensions by calliper.

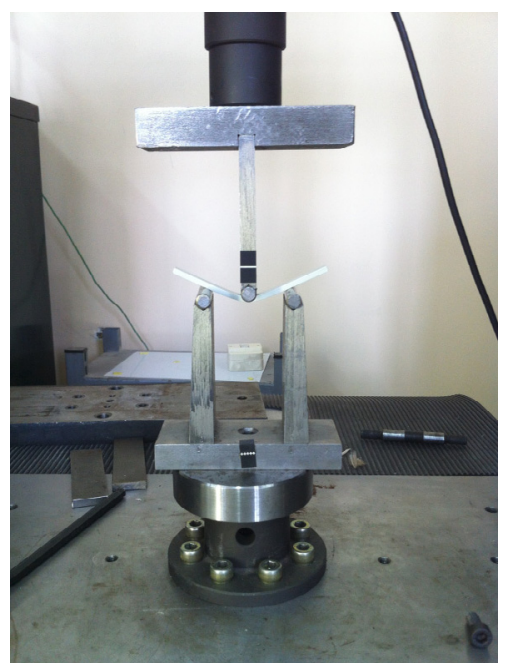

Fig. 3. Measurement of test sample during flexure testing.

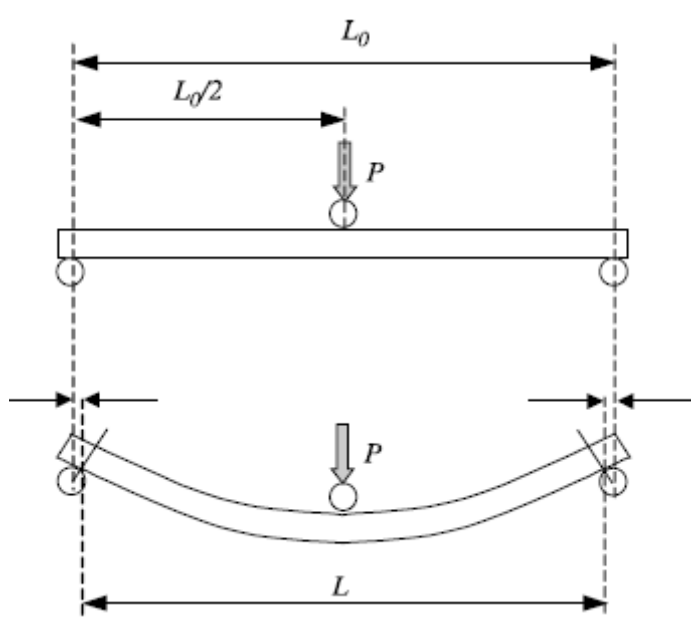

Fig. 4. Effect of deflection to the computed length.

\section{Results and discussion}

Experiments were made for two different geometries to investigate the effects of length to the uncertainty. A small difference was noticed for these different samples.

AG-IC Floor Type tensile test equipment with $50 \mathrm{kN}$ capacity was used in mechanical properties laboratory. For the testing specimen geometries $1 \mathrm{kN}$ capacity load cell was used. Deflections of points were recorded by two 
Table 2. Calculations of sensitivity coefficients and uncertainties for the samples.

\begin{tabular}{cccccccccc}
\hline & $\begin{array}{c}C F, \\
{\left[1 / \mathrm{mm}^{2}\right]}\end{array}$ & $\begin{array}{c}C A, \\
{\left[\mathrm{~N}^{2} / \mathrm{mm}^{4}\right]}\end{array}$ & $\begin{array}{c}C a c, \\
{[\mathrm{~mm}]}\end{array}$ & $\begin{array}{c}u \sigma \\
{\left[\mathrm{N} / \mathrm{mm}^{2}\right]}\end{array}$ & $\begin{array}{c}u F, \\
{[\mathrm{~N}]}\end{array}$ & $\begin{array}{c}u A, \\
{\left[\mathrm{~mm}^{2}\right]}\end{array}$ & $\begin{array}{c}u A c \\
{\left[\mathrm{~mm}^{2}\right]}\end{array}$ & $\begin{array}{c}u_{\text {test }} \\
{\left[\mathrm{N} / \mathrm{mm}^{2}\right]}\end{array}$ & $\begin{array}{c}U_{\text {exp }} \\
{[\mathrm{mm}]}\end{array}$ \\
\hline ABS-1 & 0.00004 & -0.32 & 34.1 & 2.24 & 47.5 & 20.3 & 0.021 & 1.87 & 2.72 \\
ABS-2 & 0.000031 & -0.30 & 34.1 & 2.10 & 35.2 & 35.4 & 0.021 & 1.41 & 2.53 \\
\hline
\end{tabular}

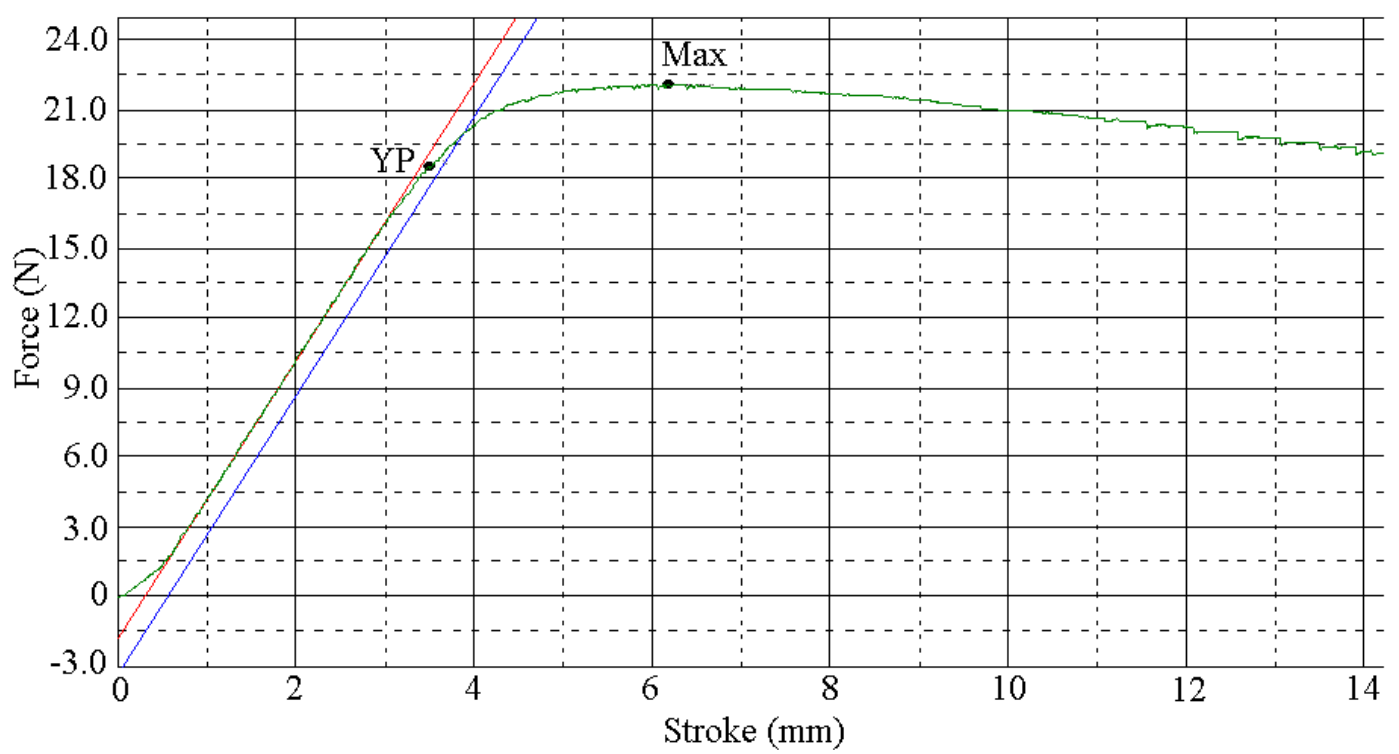

Fig. 5. Strain-stress curves for ABS-2 obtained from experiment.

cameras. To provide a reference length for the cameras a calibration bar was used.

Some values were directly taken from machine software (Fig. 5). UF is directly taken from calibration certificate of material testing machine and $U$ c-cert. was also taken from the calibration certificate of the caliper.

The average thickness of the shorter samples (ABS-1) was $2.82 \mathrm{~mm}$, the average width is $10 \mathrm{~mm}$, the average maximum force is $3052.3 \mathrm{~N}$ and the average tensile strength is $103.3 \mathrm{~N} / \mathrm{mm}^{2}$. The tensile strength and uncertainty in the result of tensile test are shown $103.3 \mathrm{~N} / \mathrm{mm}^{2} \pm 2.7 \mathrm{~N} / \mathrm{mm}^{2}$ (Tab. 2).

The average thickness of the longer samples was $3.69 \mathrm{~mm}$, the average width is $25 \mathrm{~mm}$, the average maximum force is $2651.8 \mathrm{~N}$ and the average tensile strength is $179.6 \mathrm{~N} / \mathrm{mm}^{2}$. The tensile strength and uncertainty in the result of tensile test are shown $179.6 \mathrm{~N} / \mathrm{mm}^{2} \pm 2.3 \mathrm{~N} / \mathrm{mm}^{2}$ (Tab. 2).

In literature there are a big variety of studies which investigated three point bending tests from different point of views. Quintela and Sánchez [10] studied static behavior of a three-dimensional elastic certain assumptions of compatibility, concerning to applied beam by means of mathematical computational methods and proved the existence of a unique solution for the associated three-dimensional elastic model with contact constraints when forces and symmetry properties.

Krasovskii et al. [11] studied dynamics of impact testing in bending test and proposed a computational model in order to get a general solution from equations. These equations described the process of the impact testing of specimens in concentrated bending in the presence of supports. Rvachev and Uchishvili [12] studied the formulation step of bending test. In their study the maximum deflection which is formed from rotation of the beams at the supports. As mentioned before this deflection also one of the important uncertainty source. Polyakov et al. [13] studied mathematically the deflections, local curvatures, and bending stresses, dependent on the face-layer thicknesses and transverse characteristics of the filler. These are also considered as unexpected uncertainty sources in conventional three point bending test. Huang et al. [14] studied on obtaining dynamic stress intensity factor from the relationship of $J$ integral, Crack Open Mouth Displacement with the Dynamic Stress Intensity Factor.

Test sample materials are also one of the uncertainty sources as stated before. Mujika [15] studied of the difference of flexural modulus that are obtained from threepoint and four-point bending tests for the same specimen. Mujika analysed the effect of the variation of the support span and the load span caused by the variation of the contact zone between the specimen and support and load rollers that are also considered to be uncertainty sources.

As a result of developing technology there are many newly developed materials. Three point bending test is needed for determining their mechanical properties. Giglio et al. [16] studied sandwich panels with Al skins and Nomex ${ }^{\mathrm{TM}}$ Honeycomb material behavior while three point bending test by comparing numerical results and experimental data. They considered influence of various 
parameters like friction and relative position of the puncher which in their numerical investigations. Kimura et al. [17] used three-point bending tests to determine fracture strengths their millimeter-scale tourmaline and epidote samples by means of using different size-effect models for selecting the most appropriate size of their materials test sample. All of these studies aimed finding flexural quantities of materials by means of three point bending tests by numerical modeling or experimental studies to determine effective parameters to the measurement results and accuracy during the test procedure.

\section{Conclusion}

An analysis of the uncertainty sources incorporated during measurements of the flexure strength of the ABS material has been performed. Although not all sources of uncertainty have been investigated in detail, calculable sources such as dimensions of tested bars, cross-section, uncertainty of calipers and applied forces and so on are added into measurement uncertainty of flexure strength results. Examples illustrate the importance of uncertainty sources relevant to the variability of the parameters measured from a series of tests bars in same group of ABS material. According to the prior studies [18] this study assumes that the error terms don't depend on the material properties or load span used. Thickness and length of the specimen were determined to be the most effective uncertainty sources. The relationship between the measurement uncertainty and uncertainty components for different lengths were identified, which would help both reduce the measurement cost and improve the measurement productivity. These results also will provide the user and producers to select more appropriate material of their product which provides more safety and functionality. As stated in literature review [10-17] there are many different kind of uncertain sources which are also have important role on combined uncertainty. In the second step of this research new kind of material and more different sized geometries are aimed to investigate as effective uncertainty components. These results could be useful for guiding the practical engineering design.

\section{References}

1. R.D. Heap, R.H. Norman, Flexural Testing of Plastics (Plastics Institute, London, 1969)

2. K.J. Stout, M. Numan Durakbasa, P. Herbert Osanna, Quality assurance - Have we specified it the wrong way round? Wear J. 266, 511-514 (2009)

3. R.J. Brown, Handbook of Polymer Testing (Marcel Dekker, New York, 1999)
4. S. Vejelis, S. Vaitkus, Investigation of Bending Modulus of Elasticity of Expanded Polystyrene (EPS) Slabs, Flexural Properties of Plastics, Mater. Sci. 12 (2006)

5. The International Organization for Standardization 178:2010 (E) Flexural Properties Testing of Plastics and Polymers

6. ISO/IEC Guide 99:2007 International vocabulary of metrology - Basic and general concepts and associated terms (VIM)

7. R.K. Leach, Fundamental Principles of Engineering Nanometrology (William Andrew, 2009)

8. EN ISO 14253-1:1998/prA1:2011; Geometrical Product Specifications (GPS) - Inspection by measurement of workpieces and measuring equipment - Part 1: Decision rules for proving conformance or non-conformance with specifications

9. T.M. Adams, A2LA Guide for Estimation of Measurement Uncertainty in Testing The American Association for Lab. Accreditation 2002, Guidance $\backslash$ G104

10. P. Quintela, M.T. Sánchez, Three-point bending tests - Part I: Mathematical study and asymptotic analysis Mathematical Methods, Appl. Sci. 34, 1211-1235 (2011)

11. A.Ya. Krasovskii, I.V. Orynyak, A.V. Naumov, V.N. Krasiko, Dynamics of the process of impact testing in concentrated bending, Report 2. Three-point bending, Strength Mater. 21, 697-702 (1989)

12. V.L. Rvachev, L.A. Uchishvili, A method to solve the problem of bending of bending a plate clamped along its contour, Sov. Appl. Mech. 4, 76-78 (1968)

13. V.A. Polyakov, I.G. Zhigun, R.P. Shlitsa, V.V. Khitrov, A refined model for three-point bending of sandwich panels. 1. Deflections and bending stresses, Mechanics Comput. Mater. 33, 526-542 (1997)

14. Yayu Huang, Xiangping Hu, Yujie Shen, Taohong Liao, Method of obtaining dynamic stress intensity factor by Measuring Crack Mouth Opening Displacement on a 3-point bending specimen, Electr. Information Eng. Mechatron. 138, 1269-1275 (2012)

15. F. Mujika, On the difference between flexural moduli obtained by three-point and four-point bending tests, Dep. Mech. Eng. 25, 214-220 (2006)

16. M. Giglio, A. Gilioli, A. Manes, Numerical investigation of a three point bending test on sandwich panels with aluminum skins and Nomex ${ }^{\mathrm{TM}}$ Honeycomb core, Comput. Mater. Sci. 56, 69-78 (2012)

17. N. Kimura, H. Awajib, Masato Okamotob, Yoshihisa Matsumurab, Toshiaki Masudaa, Fracture strength of tourmaline and epidote by three-point bending test: application to microboudin method for estimating absolute magnitude of palaeo differential stress, J. Struct. Geol. 28, 1093-1102 (2006)

18. F. Guitián, P. Quintela, M.T. Sánchez, V. Valcárcel, Three point bending tests, Part II: An improved formula for the modulus of rupture and numerical simulations, Math. Methods Appl. Sci. 34, 1254-1273 (2011) 